\title{
Gender Violence in the University: The Role of Higher Education Institutions in its Prevention
}

\section{Violencia de Género en la Universidad: El Rol de las Instituciones de Educación Superior en su Prevención}

\section{Mejía Paredes, S. Veloz Miño, and R. Saeteros Hernández}

Carrera Promoción y Cuidados de la Salud, Facultad de Salud Pública, Escuela Superior Politécnica de Chimborazo, Riobamba, Ecuador

VII International Congress of

Science, Technology,

Entrepreneurship and

Innovation (SECTEI 2020)

Corresponding Author:

M. Mejía Paredes

martha.mejia@espoch.edu.ec

Published: 26 August 2021

Production and Hosting by

Knowledge E

(c) M. Mejía Paredes et al. This article is distributed under the terms of the Creative Commons Attribution License, which permits unrestricted use and redistribution provided that the original author and source are credited.

\section{Abstract}

Talking about gender-based violence and sexual harassment at the Ecuadorian university has been considered by many as an uncomfortable subject, and for some years it has been silenced. It is only recently that this situation has become an essential topic to investigate, so that currently several universities have struggled to explore through studies the problems of gender violence, discrimination and sexual harassment in the university context. In this sense, the present study has tried to develop a review of all the investigations that have been carried out to identify cases or situations of gender violence in universities at international, national and local level, as well as to determine the role of education institutions superior in the prevention and eradication of this problem.

Keywords: gender violence, sexual harassment, university.

\section{Resumen}

Hablar de violencia de género y acoso sexual en la universidad ecuatoriana ha sido considerado por muchos como un tema incómodo por lo que durante algunos años ha permanecido silenciado. No es sino hace poco que esta situación se ha vuelto una temática imprescindible de investigar, por lo que actualmente varias universidades se han esforzado en explorar a través de estudios los problemas de violencia de género, discriminación y acoso sexual en el contexto universitario. En este sentido, el presente estudio ha pretendido desarrollar una revisión de todas las investigaciones que se han realizado para identificar los casos o situaciones de violencia de género en universidades a nivel internacional, nacional y local, así como determinar el rol de las instituciones de educación superior en la prevención y erradicación de esta problemática.

Palabras Clave: violencia de género, acoso sexual, universidad.

\section{Introducción}

En Ecuador 6 de cada 10 mujeres han experimentado algún tipo de violencia al menos S OPEN ACCESS 
Género contra las Mujeres [1]. Este tipo de violencia se presenta en todos los espacios y el contexto universitario no es la excepción, debido a las desiguales relaciones de poder existentes en este ámbito.

Numerosos estudios demuestran que muchas mujeres en la etapa universitaria (2024 años) tienen un elevado riesgo de sufrir violencia por parte de su pareja, además, la violencia durante la época de noviazgo es tres veces más frecuente que en las parejas adultas aunque menos graves, apareciendo aquí las primeras agresiones [2]. Con frecuencia las jóvenes identifican como violencia solamente las agresiones de tipo físico como golpes, empujones o ataques sexuales, mientras que los maltratos psicoemocionales muchas veces no los reconocen como tal, por tanto, no los denuncian [3].

A nivel internacional existen un sinnúmero de investigaciones sobre violencia de género en el espacio universitario, donde se muestran cifras preocupantes de violencia y agresiones sexuales, que afectan entre un 13 y 30\% a estudiantes universitarias. Además de recibir acoso o abusos por parte de compañeros o parejas sentimentales, pueden padecer situaciones de acoso sexual de profesores universitarios [4]

Lastimosamente, en Ecuador los estudios sobre violencia de género o acoso sexual en el ámbito universitario son prácticamente escasos, algunos de ellos son realizados en instituciones de educación superior específicas, pero no existen estudios a nivel nacional. Debido a esta situación, esta problemática es invisibilizada y los mecanismos adecuados para la recepción de denuncias, registro de casos y protección de las víctimas llegan a ser insuficientes [5].

Sin embargo, de acuerdo con el estudio 'Construcción de un instrumento para la medición del acoso sexual en Instituciones de Educación Superior (IES) del Ecuador' realizado en diferentes universidades, se pudo determinar que en 2017 alrededor del $15 \%$ de estudiantes mujeres reconoció haber recibido comentarios intimidatorios sobre su forma de vestir, su cuerpo o su sexualidad; el 13\% bromas de contenido sexual y miradas morbosas; y al 9\% le han intentado besar o abrazar sin consentimiento [6].

En este sentido, el presente artículo pretende realizar un análisis de algunos resultados de investigaciones sobre la existencia y características de las situaciones de violencia de género y acoso sexual en estudiantes universitarias a nivel nacional y una revisión en el contexto internacional; así como las medidas y acciones que existen en las diferentes universidades para prevenirla o detenerla, con la aplicabilidad de este tipo de medidas en las Instituciones de Educación Superior del Ecuador.

\section{Metodología}

La presente investigación se basa, inicialmente, en una revisión bibliográfica de las principales investigaciones que a nivel internacional y nacional se han desarrollado 
sobre violencia de género y acoso sexual en las universidades, considerando como punto importante el rol de las Instituciones de Educación Superior en la identificación y aplicación de medidas, políticas universitarias o protocolos de actuación frente a actos de violencia, discriminación o acoso sexual, y que contribuyen a su prevención y superación. La revisión documental se ha llevado a cabo tomando en cuenta criterios de exhaustividad y cientificidad, recurriendo a bases de datos de reconocimiento internacional en Ciencias de la Salud, Sociales y Educativas.

Las bases de datos incluidas han sido Dialnet, Redalyc, Scielo, Redib, DOAJ, Science Direct, PubMed, Scopus, utilizando como descriptores en español o inglés: violencia de género, acoso sexual en el espacio universitario.

Los criterios de inclusión fueron investigaciones originales publicadas en español o en inglés, en formato virtual, durante el periodo 2000-2019, que hayan sido publicadas en revistas especializadas o presentadas en congresos científicos y que tuvieran como objetivo el estudio de la violencia de género, discriminación o acoso sexual con muestras conformadas por estudiantes de diversas universidades del mundo con edades entre 18 y 25 años.

Por otra parte, se excluyeron investigaciones con muestras que no fueran exclusivamente constituidas por universitarios, estudios que no proporcionaran suficiente información metodológica así como aquellos trabajos que no permitieran el acceso a los textos completos o por lo menos a los datos más relevantes de la investigación respectiva.

Finalmente, se organizan y describen las investigaciones según:

1. Evidencias a nivel internacional, nacional y local sobre violencia de género y acoso sexual.

2. Factores de riesgo para la presencia de violencia de género y acoso sexual.

3. Rol de las instituciones de educación superior.

\section{Desarrollo y Discusión}

La violencia de género y específicamente la violencia contra la mujer, es considerada como un importante problema de salud pública por su creciente incidencia y mortalidad [7], y considerada por la OMS como un problema de salud de proporciones epidémicas [8]. Según los Centros para el Control y la Prevención de Enfermedades (CDC), describe 'cualquier daño físico, sexual o psicológico por parte de una pareja o cónyuge actual o anterior'. Una serie de investigaciones han demostrado importantes implicaciones negativas para la salud de las personas víctimas de este tipo de violencia, como síntomas de salud mental, uso de sustancias, lesiones físicas e infecciones por VIH/ITS [9]. 
Respecto a las situaciones relacionadas con la violencia de género se analiza a la universidad como una institución donde se genera un ambiente adverso hacia las mujeres, e indica que el acoso sexual y la misoginia se manifiestan en el día a día, entre diferentes colectivos, en los currículums académicos o en los debates de las aulas. En este contexto hay formas de subordinación y de violencia hacia las mujeres, un intento de control mediante la fuerza, la dominación o el silencio [10].

Se consideró importante el análisis de algunas temáticas relacionadas a la violencia de género en universitarias tales como evidencias a nivel internacional, nacional y local, factores de riesgo que pueden desencadenar acciones de violencia así como la respuesta de las instituciones universitarias y sus propuestas de actuación en contra de esta problemática.

\subsection{Violencia de género y acoso sexual en las universidades. Evi- dencias a nivel internacional, nacional y local}

Investigaciones relevantes a nivel internacional dan cuenta de la magnitud de la problemática en torno a la violencia de género y acoso sexual en el espacio universitario.

Las primeras investigaciones sobre esta temática han sido desarrolladas básicamente en Estados Unidos y Canadá. Algunas de ellas demostraron que un 23\% de mujeres había padecido algún tipo de abuso o situación no deseada, además, se determinó que algunas de las violaciones que se presentaron en los campus universitarios en el $54 \%$ de los casos suceden en las citas, y el $35 \%$ de chicos universitarios afirmaron que cometerían una violación si pudiesen [10]. Similares estudios demostraron que el 51\% de las jóvenes estudiantes había sufrido al menos un acto de agresión sexual desde que tenía 14 años y 83\% de ellas había sido víctima mientras estaba en la universidad. El 95\% de las mujeres que había sufrido esta situación refirió que conocía a su agresor, que era su novio, amigo o conocido y en un $38 \%$ de los casos lo había conocido el mismo día o durante la noche de la agresión [11]

Otro estudio destaca que el $27 \%$ de mujeres estudiantes desde que se matriculó en la universidad había experimentado contacto sexual no deseado, desde besos y caricias hasta relaciones sexuales orales, anales o vaginales [12]. Una investigación importante sobre violencia de género en las relaciones desiguales, mostró la existencia de acoso sexual por parte de profesores universitarios hacia los estudiantes, correlacionado variables como acoso, género y grupo étnico, determinando que un $40 \%$ de mujeres y un $28,7 \%$ de hombres había recibido acoso por parte de un profesor [13].

Del contexto europeo se destacan algunas investigaciones realizadas sobre todo en España, así tenemos que de acuerdo a un estudio realizado en la Universidad de Sevilla se registraron cifras en torno al $12 \%$ en agresiones físicas (bofetadas y puñetazos), al menos una vez, en chicas; además, declaran haber sido forzadas al contacto sexual (al 
menos una vez) el 10,4\% de estudiantes mujeres. En lo referente a comportamientos de vigilancia y ciberacoso, afirmaron recibir mayor cantidad de mensajes controladores y sufrir mayor vigilancia que los estudiantes varones [2]. Además, se puede mencionar que para analizar la realidad entorno a la violencia de género en las universidades españolas, se diseñó, probó e implementó un cuestionario a un total de 1.083 hombres y mujeres estudiantes de seis universidades de comunidades autónomas distintas (Universidad de Barcelona, Universidad de Sevilla, Universidad del País Vasco, Universidad de Murcia, Universidad de Valladolid y Universidad Jaume I), encontrando que un 52\% afirmaron que han sufrido o conocen a alguien que haya sufrido violencia de género en el ámbito universitario [14].

En la Universidad de Oviedo se investigó episodios de violencia en las relaciones de pareja en estudiantes de Enfermería, encontrando que el $86 \%$ había sufrido una o más conductas de maltrato, entre estas se encuentra la coerción en el 73\%, humillación $58 \%$, castigo emocional $42 \%$ y agresión sexual el 37\% [15].

Muñoz et al. en su investigación sobre agresión física y psicológica en estudiantes universitarios de universidades públicas y privadas de la Comunidad Autónoma de Madrid encontró que el $12 \%$ de las mujeres mencionaron que sufrieron agresiones físicas de sus parejas (cortes leves o contusiones), mientras que el 58\% refirió haber recibido agresión verbal [16].

Centrándose en el acoso sexual por parte de profesorado universitario al estudiantado, algunos estudios obtuvieron como resultado que de 525 personas encuestadas, el $40 \%$ de las mujeres y el $28,7 \%$ de los hombres habían sido acosadas y acosados por profesorado de la universidad [11].

Un estudio realizado en la Universidad de Oulu en Finlandia mostró que el 40\% de estudiantes refirió haber experimentado humillación o desprecio, el 34\% comentarios despectivos, el $23 \%$ gritos e insultos y el $17 \%$ acoso sexual y maltrato basado en género [17].

La más grande investigación realizada sobre esta temática presenta las tasas de violencia contra parejas de citas por parte de estudiantes de 31 universidades en 16 países ( 5 en Asia y Medio Oriente, 2 en Australia-Nueva Zelanda, 6 en Europa, 2 en América Latina, 16 en América del Norte). Las tasas de agresiones y lesiones se presentan para hombres y mujeres en cada una de las 31 universidades, determinando que el 29\% de los estudiantes agredieron físicamente a una pareja en los últimos 12 meses [18]. Del total de mujeres que sufrieron experiencias de coacción sexual, el 41\% de los agresores eran sus parejas, los amigos el $29 \%$ y los conocidos el $21 \%$ [14].

Igualmente se han realizado una serie de estudios a nivel de países de Latinoamérica, encontrando que el $\mathbf{8 6 . 4 \%}$ de estudiantes refirió haber sido sometido a actos de discriminación o violencia durante su estancia en la Universidad. En una investigación llevada a cabo en la Universidad de Caldas en Colombia, se pudo determinar que 
alrededor del $44 \%$ de estudiantes mujeres había sido víctima de agresión sicológica, además, refirió recibir burlas, piropos y gestos obscenos y un agresiones de tipo verbal $36 \%$, el acoso sexual y agresiones físicas se presentaron en el $5 \%$ y $3 \%$ respectivamente [19]. En relación al acoso sexual un estudio realizado en la Universidad de Manizales mostró que la mayoría de casos fueron propiciados por docentes en un $50 \%$ y en un $21,4 \%$ por parte de compañeros, y solamente un 7,1\% lo había denunciado [20].

La universidad de Pereira en Colombia realizó una investigación en 128 estudiantes encontrando que el $13 \%$ reportó la existencia de uno o más eventos de violencia sexual durante su vida universitaria. El tipo de violencia sexual más frecuente fue el acoso sexual (28 episodios), el cual fue leve o verbal en el 70\%. Hubo dos casos de violación. De las víctimas el 37\% reportaron el hecho [21].

En Chile estudios como el de Vizcarra y Póo sobre violencia de parejas en estudiantes universitarios del sur de Chile, determinó que el 57\% de estudiantes investigados reportó haber sido víctima de violencia psicológica alguna vez en su vida y un $26 \%$ violencia física [22]. Otros resultados muestran que la prevalencia de haber recibido algún tipo de violencia en sus relaciones de pareja es de $86,6 \%$ en las estudiantes mujeres, encontrándose en una mayor proporción (87.5\%) la violencia psicológica, El $6.3 \%$ refirió haber sufrido violencia sexual [23]. El estudio realizado por Lehrer (2007) en una universidad pública chilena muestra que el $17 \%$ de estudiantes informaron haber experimentado alguna forma de contacto sexual no deseado en los últimos 12 meses, mencionando que el consumo de alcohol u otras drogas fueron utilizados en la mayoría de los casos de violación o intento de violación, por la víctima (6\%), el perpetrador (9\%) o ambos (56\%) [24]. Por su parte Sandoval Obando (2019) en su estudio sobre violencia de género y discriminación arbitraria en una universidad privada chilena estudiantes declararon haber presenciado o experimentado episodios de violencia psicológica (18\%), violencia de género (6\%) y violencia sexual (1\%) [25].

En Argentina una investigación realizada en la Universidad San Martín sobre acoso sexual presentó lo siguientes hallazgos: Las mujeres declararon en la encuesta que vivieron situaciones de violencia simbólica a causa de género u orientación sexual (35\%), de violencia psicológica y verbal (18\%) y sexual en conjunto con la psicológica y verbal (12\%), el $78 \%$ de las estudiantes respondió que padeció alguna tipo de acoso sexual y $83 \%$ escuchó comentarios subidos de tono u obscenos, silbidos, gestos en el aula, pasillos u otros espacios de la Universidad, en cuanto al acoso sexual grave lo sufrió el $73 \%$ a través de llamadas telefónicas, mensajes de texto, correos electrónicos, mensajes o comentarios en Facebook referidos a su vida íntima. También aparece un alto porcentaje de mujeres (83\%) que sufrieron acoso sexual muy grave, a través de prácticas tales como tocado, forcejeado, besado sin su consentimiento. Un $67 \%$ fue citada en un aula, oficina, laboratorio u otro sitio de la Universidad, innecesariamente, para hacerles proposiciones inadecuadas y/o de tipo sexual [26]. 
Una compilación importante de evidencias sobre violencia en el noviazgo de jóvenes universitarios en México presentó los siguientes resultados relevantes: El 33.3\% de los hombres manifestaron haber agredido alguna vez a su pareja, 47\% manifestó estar en relaciones con algún tipo de violencia, uno de cada cuatro estudiantes manifestó haber sido violentado/a alguna vez en sus relaciones afectivas, el $38 \%$ reportó haber sufrido agresiones verbales, 36\% agresiones físicas, $7 \%$ agresiones con arma y $19 \%$ violencia sexual [27]. Otro estudio señaló que el $60.59 \%$ de las estudiantes indicaron que han sido agredidas por hombres dentro de la universidad. En ella la violencia psicológica fue la más señalada (77.82\%), seguida de la física (45.89\%), la económica (45.48\%) y la sexual (27.16\%), además, dentro de los actos de violencia sexual que experimenta el alumnado, el acoso sexual fue el más mencionado con el $23.69 \%$ de las mujeres [28].

En nuestro país, estudios sobre violencia de género y acoso sexual hacia las mujeres son prácticamente escasos, sin embargo, se considera rescatable el estudio 'Construcción de un instrumento para la medición del acoso sexual en Instituciones de Educación Superior (IES) del Ecuador' realizado en diferentes universidades, de acuerdo al cual se pudo determinar que en 2017 alrededor del 15\% de estudiantes mujeres reconoció haber recibido comentarios intimidatorios sobre su forma de vestir, su cuerpo o su sexualidad; el $13 \%$ bromas de contenido sexual y miradas morbosas; y al $9 \%$ le han intentado besar o abrazar sin consentimiento [6].

Por su parte, Barredo Ibáñez en su estudio 'La violencia de género en Ecuador: un estudio sobre los universitarios' identificó que cinco de cada diez estudiantes investigados opinaban que la manera de vestir o el comportamiento de una mujer podía incitar a la violación, 52,8\% eran hombres. Alrededor de dos de cada diez universitarios aseguraban haber ejercido alguna vez violencia contra sus parejas [29].

Un estudio sobre sexismo en tres universidades ecuatorianas demostró que el 41.3\% de los participantes ha ejercido violencia física media, mientras que un $36.4 \%$ la ha sufrido. En cuanto a la violencia física grave, fue ejercida por un $7.4 \%$ y sufrida por un $10.5 \%$ de los encuestados. Respecto a la violencia psicológica fue ejercida por un $15.2 \%$ y sufrida por un $13.8 \%$ [30].

En la investigación de Guarderas et al. sobre acoso sexual en universitarias mostró que el $27 \%$ de estudiantes había sido víctima de acoso sexual. El $56 \%$ de las situaciones fueron realizadas por sus pares y el 75\% las protagonizaron varones [31].

Además, se pueden encontrar investigaciones realizadas por universidades como un esfuerzo por dilucidar la problemática de la violencia de género y acoso sexual, es así que a nivel local se evidencian estudios realizados por la Escuela Superior Politécnica de Chimborazo en donde se observó que el mayor porcentaje de estudiantes refiere haber recibido violencia por parte de otro estudiante, tanto física (15\%), como psicológica (11\%). Un menor porcentaje (8\%) menciona haber sufrido maltrato por parte de su pareja 
(bofetadas, golpes, heridas) y el 5\% reporta violencia psicológica, por parte de algún docente (desprecio, desvalorización y rechazo) [32].

\subsection{Factores de riesgo para la violencia de género en el ámbito universitario}

Existen investigaciones dirigidas a estudiar los factores asociados a la violencia de género, sobre todo en la población juvenil en quienes se encuentra normalizada la situación de violencia, convirtiéndose en algo sumamente preocupante debido a que es común ver comportamientos violentos en su forma de relacionarse.

Diversos estudios manifiestan que las conductas ejercidas desde muy jóvenes en el entorno de la pareja puede condicionar su forma de vivir la relación en etapas posteriores, pudiendo actuar como precursor de situaciones de violencia más grave en la edad adulta [33].

\subsubsection{Características demográficas}

El riesgo de violencia es mayor entre chicos que pertenecen a un nivel socioeconómico bajo, que tienen un menor nivel educativo, que son inmigrantes no adaptados y que viven en un entorno urbano [34].

\subsubsection{Creencias y actitudes en torno a la violencia de género}

Las creencias y actitudes más tolerantes hacia la violencia contra las mujeres constituyen uno de los principales factores de riesgo socioculturales para la presencia de esta forma de maltrato. Es así que la mayor parte de investigaciones sobre esta temática atribuyen a cuestiones estereotipadas de género, encontrando una mayor tendencia de los varones a culpar a las mujeres víctimas por la violencia sufrida. En definitiva, se ha detectado que los varones y las personas con actitudes de rol de género tradicionales tenderían a presentar en mayor medida actitudes positivas hacia la violencia contra las mujeres en la pareja [35].

En los sistemas sociales patriarcales subsiste la creencia de que la violencia es una forma bajo la cual los fuertes pueden dominar a los débiles y se tiene evidencia de que la universidad sigue estando dominada por los hombres, los cuales ejercen este predominio a través de diferentes formas de misoginia y de discriminación [36].

La aparición de las actitudes sexistas cada vez a más temprana edad configura un marco de relaciones idóneo para el desarrollo de comportamientos de abuso y violencia en las relaciones de pareja que establecen adolescentes y jóvenes Este sexismo muy presente en las y los estudiantes, refuerza el rol tradicional de la mujer, justificando las 
actitudes que implican violencia [30]. El sexismo es un factor cultural que forma parte de la organización social universitaria estableciendo jerarquías y privilegios entre los géneros, posicionando al modelo masculino heterosexual en la cúspide [26].

Ciertas situaciones pueden desencadenarse a partir de actitudes de ignorancia acerca de la violencia de género, y en particular del acoso sexual, por ejemplo: atribuir a la mujer la culpa de este tipo de actos por la forma en que visten, hablan, miran, caminan, bailan, beben, se arreglan; por los lugares y horas en que transitan, por las compañías que frecuentan y al naturalizar esta práctica posicionan a la mujer como objeto para la satisfacción de la supuestamente 'incontrolable pulsión sexual' de los varones [37].

\subsubsection{Características de personalidad}

En algunas ocasiones la violencia en la pareja puede estar asociada a un déficit de habilidades sociales, a celos patológicos o a trastornos de personalidad, como la psicopatía, una dependencia emocional excesiva o el trastorno límite de personalidad. Los hombres muy dependientes pueden ser violentos porque tienen horror al vacío si no dominan a su pareja o si ella les abandona [38].

Charkow y Nelson estudiaron a 178 mujeres estudiantes universitarias e identificaron violencia o coacción sexual en las citas los cuales respondían a relaciones caracterizadas por aspectos de dependencia y aceptación del abuso confundiéndolo con amor o con ideales tradicionales románticos [39].

\subsubsection{Variables psicopatológicas}

El abuso de alcohol y drogas, en la medida en que actúa como un factor desinhibidor, es una variable predictora de la violencia de pareja entre los adolescentes y jóvenes [40]. Algunas otras alteraciones psicopatológicas como la ansiedad, la depresión o el trastorno de estrés postraumático, pueden manifestarse en forma de irritabilidad y, si median actitudes negativas hacia la pareja, transformarse en conductas violentas [41].

\subsubsection{Experiencias previas de violencia}

Haber experimentado violencia en etapas de infancia facilita el desarrollo ulterior de un estilo de vida violento, tales como exposición a violencia entre los padres o ser víctima de maltrato o abuso sexual. Los chicos aprenden que la violencia es una forma de salirse con la suya y las chicas que sufrir la violencia es inevitable en la relación con los hombres [38]. 


\subsubsection{Las víctimas no identifican situaciones de violencia de género}

Un aspecto importante que hay que considerar es que muchas de las mujeres que sufren actos de violencia o acoso sexual no las consideran como tal.

Estudios han mostrado que a pesar de que algunas mujeres han sido forzadas a tener relaciones sexuales no deseadas, consideradas como una violación, las víctimas no lo aceptaron o reconocieron, incluso no lo denunciaron [11], así lo muestra un estudio en el cual de 246 mujeres que habían sufrido alguna situación de violencia de género, solamente 4 lo denunciaron ante la policía [12].

\subsection{La universidad como contexto hostil para las mujeres}

Algunos autores reconocen que las Instituciones de Educación Superior (IES) se han convertido desafortunadamente en lugares poco seguros, donde la discriminación, violencia y acoso sexual parecen ser prácticas recurrentes [42]. Es importante considerar que los centros educativos universitarios son espacios de enseñanza de los jóvenes, sin embargo, en muchas ocasiones se han presentado casos de hostigamiento y acoso que por mantenerse en silencio se han cometido una variedad de injusticias y maltratos físicos, sicológicos e incluso sexuales.

Otras investigaciones no se han centrado en identificar situaciones concretas de violencia de género en las universidades sino en analizar la universidad como una institución donde se genera un ambiente adverso hacia las mujeres y un ambiente mucho más desfavorable para las mujeres que para los hombres [11].

Con todo esto, y en medio de la desigualdad y de diversos procesos de exclusión, las jóvenes que logran ingresar a la universidad enfrentan múltiples situaciones que las hacen vulnerables a la violencia, es así que estudios realizados en países como México registran casos de agresiones físicas y de acoso sexual perpetrados por estudiantes hombres, docentes y autoridades; se observaron también formas de violencia sutiles y muchas otras que se han 'normalizado' con la complicidad de autoridades [43].

El acoso sexual y la misoginia también son formas de violencia contra las mujeres y que pueden manifestarse con normalidad en los currículos académicos, en las discusiones y debates en las aulas universitarias, siendo un mecanismo de subordinación y opresión hacia las mujeres que se vive en el día a día universitario [14].

Una situación que ha sido demostrada a través de algunos estudios es que los y las estudiantes consideran que las autoridades en las universidades no han abordado de manera adecuada esta problemática, lo que ha llevado a cierta apatía en el alumnado y a que no se denuncien incidentes de violencia o acoso sexual dentro del ámbito universitario [44]. 


\subsection{Rol de las Instituciones de Educación Superior en la imple- mentación de medidas de prevención de la violencia de género}

A raíz de las innumerables investigaciones que se han realizado en torno a la violencia de género y acoso sexual en las universidades esta situación se ha hecho más visible, haciendo que muchas de ellas adopten, diseñen o implementen una serie de políticas, acciones o protocolos encaminados a la detección y prevención de todo acto de violencia o acoso en el espacio universitario.

Algunas experiencias de universidades se mencionan a continuación:

En México, el Instituto Politécnico Nacional ha diseñado el violentómetro, además cuenta con un Programa de Gestión con perspectiva de género, que integra y pone en operación el Programa de Prevención, Atención y Sanción para Erradicar el Hostigamiento y el Acoso Sexual [36], mientras que la Universidad Nacional Autónoma de México ha establecido estrategias y propuestas dentro del Programa Universitario de Estudios de Género (PUEG); todas estas en favor de la equidad, promoviendo, elaborando y coordinando diversas investigaciones [28].

En las universidades de más prestigio internacional de Estados Unidos y Europa ya se han desarrollado programas sobre esta temática, impulsadas por investigaciones previas. En el contexto español, investigaciones se desarrollaron en la marco del Plan Nacional I+D+I financiada por el Instituto de la Mujer. En este sentido podemos mencionar la universidad de Oxford, Cambridge y Harvard, donde cuentan con una política institucional definida sobre abuso, agresión sexual u otro tipo de violencia de género, con el objetivo de lograr que la universidad sea un espacio donde resulte inaceptable una situación de ese tipo. Dentro de ella se regulan medidas disciplinarias tanto para los casos verificados de acoso sexual como pro las acusaciones malintencionadas [4].

Algunas universidades de Estados Unidos (California Institute of Technology, Massachusetts Institute of Technology, Duke University, University College London), se utilizan algunos medios como sus páginas web con información sobre temas de violencia de género y la manera de detectarlo, al igual que trípticos o guías para señalar con ejemplos situaciones de violencia de género, acoso sexual, agresión o abuso sexual.

Además se ofrece información sobre los servicios a los cuales puede acudir si se sufre algunas de estas situaciones (Princeton University, Harvard University, University of Pennsylvania, University of Manchester, Dartmouth College, University of Cambridge). En otros casos se organizan conferencias o grupos de discusión para proporcionar a estudiantes herramientas para detectar dichos actos (Columbia University). Ciertas universidades cuentan con oficinas de atención a víctimas y prevención del acoso y agresión sexual (Office of Sexual Assault Prevention and Response), así como oficinas donde denunciar estos actos de violencia, como es el caso de las universidades de 
Harvard, Yale, Stanford, Cambridge, entre otras. Muchas de ellas cuentan con políticas que permiten sancionar este tipo de sucesos [11].

En Ecuador se cuenta actualmente con un Protocolo de prevención y actuación en casos de acoso, discriminación y violencia basada en género y orientación sexual en las instituciones de educación superior, desarrollado por la Secretaría de Educación Superior, Ciencia, Tecnología e Innovación (SENESCYT) y la Red de Educación Superior y Género (RESG) coordinada por SENESCYT, considerando la necesidad de apoyar a las instituciones de educación superior (IES) en el abordaje del tema de acoso, discriminación y violencia sexo-genérica de manera conjunta con Acción Ciudadana por la Democracia y Desarrollo (ACDemocracia) y CARE Ecuador en el marco del proyecto Mujeres con Voz, cofinanciado por la Unión Europea [6].

\section{Conclusiones}

Las investigaciones sobre violencia de género en el contexto universitario ha experimentado un gran crecimiento a lo largo de los últimos 10 años, mostrando una problemática cada vez mayor, la cual requiere de acciones conjuntas entre el Estado y las Instituciones de Educación Superior para lograr su prevención y erradicación.

Luego de una revisión de estudios realizados por universidades a nivel internacional y nacional se puede determinar que existe un mayor número de investigaciones en el contexto internacional especialmente a nivel de Estados Unidos y Europa. A nivel Latinoamericano la mayor parte de estudios se encuentran sobre todo en México, Chile y Colombia.

En Ecuador las investigaciones sobre esta temática aún continúan siendo escasas.

Tras la revisión realizada se pudo observar porcentajes importantes de estudiantes universitarias víctimas de violencia de género y acoso sexual, perpetradas por sus parejas, compañeros o profesores. La violencia psicológica o emocional es el tipo de violencia que en mayor medida refieren las estudiantes investigadas.

En cuanto a los factores de riesgo se pudo determinar que estos actos se explican por valores desiguales desde la infancia, los modelos patriarcales en los cuales lo masculino tiene más valor que lo femenino fomenta en mayor medida acciones violentas hacia las mujeres.

Se ha evidenciado la existencia de medidas en muchas universidades que contribuyen a construir un ambiente donde estas agresiones son más fáciles de identificar favoreciendo su denuncia, sin embargo se considera que aún existe mucho por hacer, sobre todo en nuestro país para erradicar por completo este grave problema de salud pública. 
En este sentido, como una implicación práctica o recomendación luego de hacer una revisión sistemática sobre acciones realizadas a nivel internacional para prevenir la violencia de género en las universidades, se considera que las instituciones de educación superior en el Ecuador y sobre todo en Chimborazo, en base a las experiencias en prevención y erradicación de la violencia en universidades alrededor del mundo, deberían adoptar y experimentar estrategias que han dado buenos resultados, con la finalidad de luchar contra esta grave problemática en estos espacios educativos.

\section{References}

[1] Instituto Nacional de Estadísticas y Censos. Encuesta Nacional de Relaciones Familiares y Violencia de Género contra las mujeres. 2010 IEEE International Conference on Wireless Communications, Networking and Information Security, WCNIS 2010. Quito; 2010.

[2] García-Carpintero MÁ, Rodríguez-Santero J, Porcel-Gálvez AM. Diseño y validación de la escala para la detección de violencia en el noviazgo en jóvenes en la Universidad de Sevilla. Gac Sanit. 2018;

[3] Cortés AML, Molleda CB, Rodríguez-Franco L, Galaz MF, Ramiro- Sánchez T, Rodríguez Díaz FJ. Unperceived dating violence among Mexican students. Int J Clin Heal Psychol. 2014;

[4] Valls CR. Guía de prevención y atención de la violencia de género en las universidades. Vol. 5. 2008.

[5] Wambra Medio Digital Comunitario. Wambra Medio Digital Comunitario. Universidades Libres de Violencia de Género; 2018. Available from: https://wambra.ec/ universidades-libres-de-violencia-de-genero/

[6] Secretaría de Educación Superior C y T e I. Protocolo de prevención y actuación en casos de acoso, discriminación y violencia basada en género y orientación sexual en instituciones de educación superior. 2018.

[7] Ruiz-Pérez I, Blanco-Prieto P, Vives-Cases C. Violencia contra la mujer en la pareja: determinantes y respuestas sociosanitarias. Gac Sanit. 2004;18(Supl.2):4-12.

[8] World Health Organization. Global and regional estimates of violence against women: prevalence and health effects of intimate partner violence and non-partner sexual violence. WHO; 2013.

[9] Willie TC, Kershaw TS. An ecological analysis of gender inequality and intimate partner violence in the United States. Prev Med (Baltim). 2019;118:257-63.

[10] Fernández LR, Roldán SM. Violencia de género en las universidades: Invesstigaciones y Medidas para prevenirla. World. 2010;1:202-19.

[11] Ródenas CA, Alonso Olea MJ, Alcatud PM, Roldán SM. Violencia de género. Rev Interuniv Pedagog Soc. 2009;9(16):85-94. 
[12] Gross AM, Winslett A, Roberts M, Gohm CL. An examination of sexual violence against college women. Violence Against Women. 2006;12(3):288-300.

[13] Kalof L, Eby KK, Matheson JL, Kroska RJ. The influence of race and gender on student self-reports of sexual harassment by college professors. Gend Soc. 2001;15(2):282302.

[14] Puigvert L. Investigación sobre la violencia de género en las universidades: evidencias empíricas y contribuciones para su superación. Rev la Asoc Sociol la Educ RASE. 2008;3(2009):369-75.

[15] Díaz VG, Feito AF, Díaz FJR, González ML, Díaz MDP, Pérez AL. Violencia de género en estudiantes de enfermería durante sus relaciones de noviazgo. Aten Primaria. 2013;

[16] Rivas MJ, Gómez JLG, O’Leary KD, Lozano PG. Agresión física y psicológica en las relaciones de noviazgo en universitarios españoles. Psicothema. 2007;19(1):102-7.

[17] Rautio A, Sunnari V, Nuutinen M, Laitala M. Mistreatment of university students most common during medical studies. BMC Med Educ. 2005;5:36.

[18] Straus M. Prevalence of violence against dating partners by male and female university students worldwide. Violence Against Women. 2004;10:790-811.

[19] Moreno-Cubillos CL, Sepúlveda-Gallego LE. Discriminación y violencia contra los estudiantes de medicina de la Universidad de Caldas. Investig en Educ Médica. 2013;2(5):37-41.

[20] Castaño J, González E, Guzmán J et al. Acoso sexual en la comunidad estudiantil de la universidad de Manizales (Colombia). Univ Manizales Fac Med Cent Investig. 2009;17. Available from: http://ridum.umanizales.edu.co: 8080/xmlui/bitstream/handle/6789/1414/AcosoSexualEnlaUniversidaddeManizales, corrmartha.pdf?sequence $=1$

[21] Hinojosa-Millán S, Vallejo-Rodríguez DC, Gallo-Gómez YN, Liscano-Fierro LN, Gómez-Ossa R. Prevalencia de violencia sexual en estudiantes de la Universidad Tecnológica de Pereira, Colombia, 2010. Rev Colomb Obstet Ginecol. 2013;64(1):216.

[22] Póo AM, Vizcarra MB. Violencia de pareja en jóvenes universitarios. Terapia Psicologica. 2008;26:81-8.

[23] Muñoz RV, Moraga SE, Tregear CR, Burgos AV, Peña AV. Perpetración y victimización de la violencia en relaciones de parejas en jóvenes que cursan educación superior en la ciudad de Osorno, Chile. P. 2015;14(40):489-508.

[24] Lehrer JA, Lehrer VL, Lehrer EL, Oyarzún PB. Prevalence of and risk factors for sexual victimization in college women in Chile. Int Fam Plan Perspect. 2007;33(4):168- 75.

[25] Obando ES, Peña JC. Violencia de género y discriminación arbitraria en una universidad privada chilena: Una exploración inicial. Sophia. 2019;15(2):55-70. 
[26] Laba V. Lo personal es política universitaria: Incumbencias de las universidades nacionales frente al acoso sexual. La aljaba. 2017;21.

[27] Rojas-Solís JL. Violencia en el Noviazgo de Universitarios en México: Una Revisión. Rev Int Psicol. 2013;12(02):1-32.

[28] Zamudio-Sánchez FJ, Andrade-Barrera MA, Arana-Ovalle R, Alvarado-Segura AA. Violencia de género sobre estudiantes universitarios (as). Convergencia. 2017;24(75):133-57.

[29] Ibáñez DB. La violencia de género em Ecuador: Un estudio sobre los universitarios. Rev Estud Fem. 2017;25(3):1313-27.

[30] Boira S, Chilet-Rosell E, Jaramillo-Quiroz S, Reinoso J. Sexismo, pensamientos distorsionados y violencia en las relaciones de pareja en estudiantes universitarios

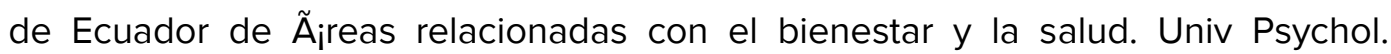
2017;16:30-41.

[31] Guarderas P, Larrea M de L, Cuvi J et al. Acoso sexual en las universidades ecuatorianas: validez de contenido de un instrumento de mediciâltextthreesuperiorn. ALTERIDAD Rev Educ. 2018;13:214-26. http://scielo.senescyt.gob.ec/scielo.php? script=sci_arttext\&pid=S1390-86422018000200214\&nrm=iso

[32] Violeta K, Avalos S, Villavicencio C et al. Discriminación y violencia de género en estudiantes politécnicos en Chimborazo - Ecuador. Cienc Digit. 2019;3:104-14.

[33] Adam A. Una revisión sobre violencia de género. Todo un género de duda. Gac Int ciencias forenses. 2013;(9):23-31.

[34] Rennison CM, Welchans S. Bureau of Justice Statistics: Intimate Partner Violence. Bur Justice Stat. 2002;1-11.

[35] Pérez VAF, Fiol EB, Palmer MCR, Espinosa GT, Guzmán CN. La violencia contra las mujeres en la pareja: Creencias y actitudes en estudiantes universitarios/as. Psicothema. 2006;18(3):359-66.

[36] Herrera CAH, García MJ, Tapia EG. La percepción del hostigamiento y acoso sexual en mujeres estudiantes en dos instituciones de educación superior. Rev la Educ Super. 2015;44(176):63-82.

[37] Mingo A, Moreno H. El ocioso intento de tapar el sol con un dedo: Violencia de género en la universidad. Perfiles Educ. 2015;

[38] González-Ortega I, Echeburúa E, De Corral P. Variables significativas en las relaciones violentas en parejas jóvenes: Una revisión. Psicol Conductual. 2008;16(2):207-25.

[39] Charkow WB, Nelson ES. Relationship dependency, dating violence, and scripts of female college students. J Coll Couns. 2000;3(1):17-28.

[40] Gil-González D, Vives-Cases C, Álvarez-Dardet C, Latour-Pérez J. Alcohol and intimate partner violence: Do we have enough information to act? Eur J Public Health. 2006;16(3):278-84. 
[41] O'Leary KD, Slep A, O'Leary S. Multivariate models of men's and women's partner aggression. J Consult Clin Psychol. 2007;75:752-64.

[42] Smit D, Plessis V. Sexual harassment in the education sector. PER Potchefstroomse Elektron Regsbl. 2011;14:172-217.

[43] Bermúdez-Urbina FM. Aquí los maestros no pegan porque ya no se acostumbra. Expresiones de la violencia hacia las mujeres en la universidad de ciencias y artes de chiapas. Península. 2014.

[44] Bryant SA, Spencer GA. University students' attitudes about attributing blame in domestic violence. J Fam Violence. 2003;18(6):369-76. 